\title{
Encapsulated nanodiamonds in smart microgels toward self-assembled diamond nanoarrays.
}

By Hugues A. Girard, *a Pierre Benayoun ${ }^{\mathrm{a}}$, Candice Blin ${ }^{\mathrm{a}}$, Adeline Trouvé ${ }^{\mathrm{a}}$, Céline Gesset ${ }^{\mathrm{a}}$, Jean-Charles Arnault ${ }^{\mathrm{a}}$ and Philippe Bergonzo ${ }^{\mathrm{a}}$

${ }^{a}$ CEA LIST, Diamond Sensors Laboratory, CEA-Tech, F-91191 Gif-sur-Yvette, France E-mail: hugues.girard@cea.fr

Keywords: nanodiamonds, pNIPAM, smart microgels, self assembly, 2D arrays

\section{Abstract:}

We report on the successful attempt to synthesize thermoresponsive nanodiamonds hybrid microgels through a straightforward method. Resulting microgels exhibit smart properties, as the reversible collapsing of the structures when raising the temperature, with embedded nanodiamonds. Thanks to the colloidal and smart properties of the microgels, nanoscaled 2D self-assembled arrays of nanodiamonds with varying inter-particle distances were realized, without any lithographic processes, as an illustration of the promising properties of these novel diamond-based microgels. Such assemblies can constitute a first step toward a bottomup elaboration of innovative diamond-based devices.

\section{Introduction}

Among "hard" nanoparticles, nanodiamonds (NDs) have become during the last decade a very promising tool for biological and technological applications, as they combine most of the physical and chemical outstanding properties of the bulk material, at the nanometric scale. For instance, they exhibit remarkable luminescence properties with emission of great stability and 
of high quantum yield which originate from color centers, as Nitrogen-Vacancy centers[1] emitting in far-red/near infrared, perfectly adapted for biological labeling [2]. Such N-V centers in nanodiamonds are also widely used as single photon emitters for quantum application, where the small size of the individual diamond crystals helps to increase the photon collection efficiency [3]. They have already been incorporated into photonic [4] and plasmonic nanostructures, or used as the apex of an AFM tip toward scanning probe magnetometry [5]. If their diamond cores exhibit the chemical and mechanical inertness of the bulk material, their surface can be widely functionalized through the carbon-related groups present on their surface: carboxylic acids, alcohols or alkenes provided by wet chemistry [6], annealing $[7,8]$ or plasma treatments $[9,10]$ act as starting points toward amidation, silanization or carbon-carbon couplings. Numerous chemical moities have thus already been successfully conjugated with NDs, toward biological uses as nucleic acids[11,12], protein $[6,13]$, genes $[11,12]$, chemotherapeutic drugs [14-18] or toward catalysis purposes[19].

Besides their use as luminescent probe with versatile surface chemistry, increasing interest has also been recently devoted to NDs in the form of thin layers. This research field has remarkably been boosted over the last years, notably because NDs can serve as seeds to promote the growth of diamond layers by Chemical Vapor Deposition (CVD) [20]. This socalled "nanoseeding" relies on the deposition of NDs as monolayers onto suitable substrates (silicon, quartz, metals), with ideally densities above $10^{11}$ particles.cm ${ }^{-2}$. Several approaches have been reported in the literature, like the dispersion by spin-coating of NDs suspended in adapted solvents[21], the self-adhesion of NDs assisted by ultrasonic agitation[20,22], or the electrostatic grafting of NDs onto a substrate driven by their surface chemistry[23,24]. By this mean, ultrathin polycrystalline diamond layers with thickness below $100 \mathrm{~nm}$ can be grown onto flat or even 3D substrates [25]. In some cases, the CVD growth is even not necessary, as NDs thin layers can already mimic some of the main advantages of nanocrystalline diamond 
layers, as their versatile surface chemistry, their chemical resilience, their biocompatibility or their optical properties, without inherent drawbacks of CVD grown layers, technologically limited in terms of size and processing temperature. For instance, Chevallier et al. reported on functionalized NDs multilayer deposited onto Surface Acoustic Wave (SAW) transducer toward enhanced sensing properties[26]. For biological purpose, highly biocompatible NDs thin layers were integrated with therapeutic molecules to realize localized drug-delivery devices[27], or used to promote the formation of functional neuronal networks [28]. In electrochemical field, Foord and coworkers reported on NDs deposited onto electrodes toward improvement of Pt particles electrodeposition [29] or enhanced catalytic properties through redox reactions occurring at the ND surface [30]. NDs layers can also be used as hard mask for etching processes, when randomly dispersed onto a substrate [31].

All these applications developed from NDs thin layers rely on well-mastered techniques to disperse them onto a substrate. Depending on the targeted properties of the film, NDs can be now deposited as monolayer or multilayers up to micrometric thickness. However, if very high densities can be achieved, the spatial ordering of the deposition still remains uncontrolled. Formation of NDs arrays still requires lithographic and etching post-processes. To our knowledge, no self-ordering of NDs has been yet reported in the literature, while selfassembly into $2 \mathrm{D}$ or even $3 \mathrm{D}$ arrays is known to spontaneously occur for some "hard" or "soft" nanoparticles. So-called "colloidal lithography", based on the self-assembly of colloids enables simple, fast and cost efficient production of 2D-ordered arrays, without the use of complex equipment. For instance, long range order nanocrystal superlattices or colloid crystals were synthesized from colloid suspensions of metallic [32] or oxides [33] nanoparticles and various polymeric beads [34], mainly toward photonic, plasmonic or catalysis applications. 
Among the polymeric beads able to self-assemble themselves in $2 \mathrm{D}$ arrays[35,36], microgels exhibit serious advantages and have been thoroughly investigated during the last decade [3741]. They are able to undergo conformational changes in response to external stimuli as temperature[42], $\mathrm{pH}[43]$ or ionic strenght[44], which enable their self-assemblies in both close-packed or periodic loosely packed arrays [35,45], with tunable separation distances, key parameter for photonic, nanofuidic devices[46], biosensors or biomedical applications[47]. Furthermore, it has been shown that hybrids systems can be designed, by combining microgels and nanoparticles[48]. Resulting composite material exhibits the swelling properties of the polymer matrix and the optical, magnetic or catalytic properties of the inorganic particle. In the last decade, such composite structures have been investigated and hybrid systems hosting, for instance, magnetic iron oxide[49], gold[50,51], silica nanoparticles[52] and quantum dots[53] have already been reported in the literature[54]. By this mean, 2D-organized arrays were synthesized, for instance with hybrid particles containing up to $20 \% \mathrm{wt} \%$ of $\mathrm{ZnS}$, in which interparticles distance can be controlled[55], or 3D nanocrystal with gold hybrids with tunable spacings between gold cores ranging from 50 to $500 \mathrm{~nm}$.

There is no example of hybrid smart microgels hosting NDs in the literature. Barras et al. recently reported on surface functionalization of NDs with pNIPAM[56], but apparently the study was led to validate a new grafting route using dopamine derivatives and no microgels were shown. Encapsulation of NDs in smart microgels could be very promising for applied or basic researches, and may offer new opportunities for NDs-based devices. Using the self assembly properties of the microgels, it would give access to controlled spatial deposition of NDs onto substrates, not accessible so far without cost and time consuming lithographic processes. In this study, we choose to work with pNIPAM microgels, based on the monomer N-isopropylacrylamide (NIPAM), first prepared by Pelton and Chibante in 1986[57], which is 
the most widely studied water-swellable microgel systems[58]. We thus propose to report on the synthesis of pNIPAM/NDs hybrid system, fully characterized by DLS, Zeta measurements, and SEM analysis. Furthermore, as an illustration of the new possibilities given by the pNIPAM encapsulation, and thanks to the colloidal properties of the microgels, driven self assemblies into close-packed or loosely-packed 2D arrays of pNIPAM/NDs hybrids were realized, exhibiting 2D organization on large scale of NDs.

\section{Experimental}

Materials \& Chemicals

High Pressure High Temperature (HPHT) nanodiamonds (NDs) were purchased from Van Moppes (Syndia ${ }^{\circledR}$ SYP 0-0.02: ND30 and Syndia ${ }^{\circledR}$ SYP 0-0.1: ND100). The NIPAM Nisopropylacrylamide (NIPAM, 97\%), the Potassium Persulfate (KPS, 99.99\%), the Sodium Hydroxide $(\mathrm{NaOH},>97 \%)$, the $\mathrm{N}, \mathrm{N}-$ methylenebisacrylamide $(\mathrm{BIS},>99.5 \%)$ were purchased from Sigma-Aldrich, and used as-received. Ultrapure water with a resistivity of $18.2 \mathrm{M} \Omega . \mathrm{cm}$ $\left(25^{\circ} \mathrm{C}\right)$ was used in all experiments.

\section{Preparation of microgels}

The microgel preparation was based on the procedure described by Pelton and Chibante[57]. In this work, N-isopropylacrylamide (NIPAM) was used as the main monomer, N,N'methylene-bis-acrylamide as a cross-linker (BIS) and potassium persulfate (KPS) as an initiator. The polymerization experiments were carried out in a reaction vessel equipped with a magnetic stirrer. $0.681 \mathrm{~g}\left(6.10^{-3} \mathrm{~mol}\right)$ of NIPAM and $0.094 \mathrm{~g}\left(6.10^{-4} \mathrm{~mol}\right)$ of BIS were introduced in $60 \mathrm{~mL}$ of degassed ultrapure water. For synthesis with nanodiamonds, NDs were directly introduced in the $60 \mathrm{~mL}$ of water at a low concentration (see table 1). To remove the oxygen, the solution was vigorously stirred for $20 \mathrm{~min}$ in the presence of nitrogen flow. Then the temperature was raised to $70^{\circ} \mathrm{C}$ and $0.6 \mathrm{~mL}$ of KPS solution at $0.1 \mathrm{M}\left(6.10^{-5}\right.$ mol) was added to initiate polymerization. When light opalescence appeared, the stirring 
speed was lowered to prevent the flocculation, and the reaction was left to proceed for $3 \mathrm{~h}$. All the microgel particles were then washed by several cycles of centrifugation-redispersion using ultrapure water.

\section{Preparation of $2 D$ microspheres arrays}

2D microspheres arrays were realized by dip-coating deposition (KSV NIMA) onto $2 \times 1 \mathrm{~cm}^{2}$ silicon substrate, at a vertical speed of ca. $15 \mu \mathrm{m} / \mathrm{sec}$, using a suspension of ND30 hybrid microgel $(50 \mathrm{mg} / \mathrm{mL})$. The deposition process was performed in a thermostatic bath.

\section{Plasma exposure}

MPCVD parameters were tuned with a pressure of $40 \mathrm{mBar}$, a $\mathrm{CH}_{4} / \mathrm{H}_{2}$ ratio of $1 / 99$. The microwave power was adjusted to $1.1 \mathrm{~kW}$ in order to reach a temperature of $750{ }^{\circ} \mathrm{C}$ after 10 min measured in situ using an optical pyrometer (IRCON MODLINE 3 bi-chromatic).

\section{Characterization}

Hydrodynamic diameters and zeta potentials of the particles were measured using dynamic light scattering (DLS) by a Malvern NanoZS at $173^{\circ}$ angle. All experiments were performed with the manufacturer calibration procedures. The average value of at least five measurements was taken at a given condition. For temperature trends, samples were equilibrated for 2 minutes before measurement.

Electron microscopy imaging was performed using an in-lens Field Emission Scanning Electron Microscope ZEISS Supra-40, operating with an acceleration voltage of $1 \mathrm{kV}$.

\section{Results and discussion}

\subsection{Synthesis of NDs hybrids microgels}

High Pressure High Temperature (HPHT) NDs were used in this study, exhibiting a mean diameter of $30 \mathrm{~nm}$ (ND30). Their surface chemistry, mainly composed by carboxylic groups 
[23], exhibits a negative zeta potential of $-45 \mathrm{mV}$ at neutral $\mathrm{pH}$ and room temperature. NDs suspended in a colloidal solution were introduced directly in the aqueous phase of the reaction mixture, at a concentration of $50 \mu \mathrm{g} / \mathrm{mL}$, before the addition of the other reagents, without any further modification. After synthesis, the resulting microgels were separated and thoroughly washed by successive centrifugation/redispersion cycles in pure water. Diluted suspensions were then characterized by DLS measurements as shown on Fig. 1. For comparison, Fig. 1 also reports DLS measurements of ND30 colloidal suspension and "pure" microspheres dispersed in water.

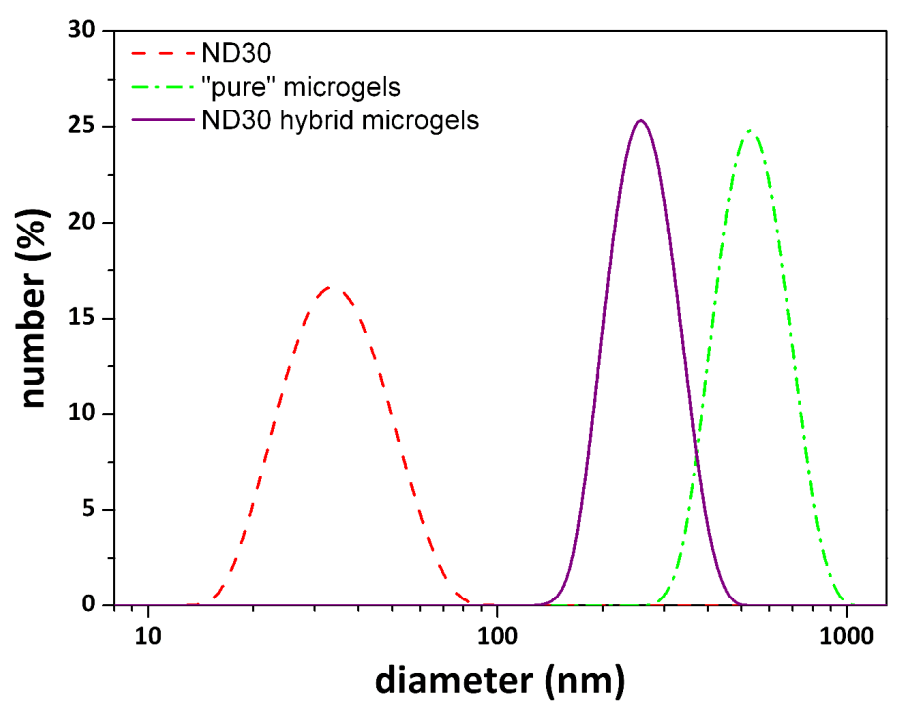

Fig.1 Hydrodynamic diameter distribution of as received ND30, "pure" microgels and ND30 hybrid microgels, recorded at $20^{\circ} \mathrm{C}$.

As-received ND30 suspension exhibits hydrodynamic diameters centered on $30 \mathrm{~nm}$. The width of the size distribution peak and its associated polydispersity index (0.2) reveals that the suspension is characterized by a scattered size distribution, coming from the production method (milling). By contrast, "pure" pNIPAM microgels exhibit a narrower size distribution peak, with a very low polydispersity index below 0.05 , reflecting a quasi-monodisperse suspension. When NDs are added to the synthesis, resulting particles exhibit a smaller 
diameter than "pure" microgels. From $531 \mathrm{~nm}$ for pNIPAM microgels, the hydrodynamic diameter decreases to $255 \mathrm{~nm}$ when NDs were added to the synthesis. Note that besides a chemical effect leading to a size reduction of the hybrid objects, a convoluted effect of the scattering of both diamond core and polymeric shell may also affect DLS measurements. However, the polydispersity index remains very low, still below 0.05 . The fact that the polydispersity index remains at a very low value for hybrid microgels highlights the efficiency of the cleaning process. An inefficient rinsing, resulting in free NDs among the microspheres, would have led to a higher polydispersity index. Furthermore, an adsorption of NDs at the surface of the microgels is not consistent with the present observations, as it would have, at the opposite, induced an increase of their size. Introduction of NDs in the reaction mixture is however clearly at the origin of a severe modification of the pNIPAM microgels structure, illustrated by the diameter decrease.

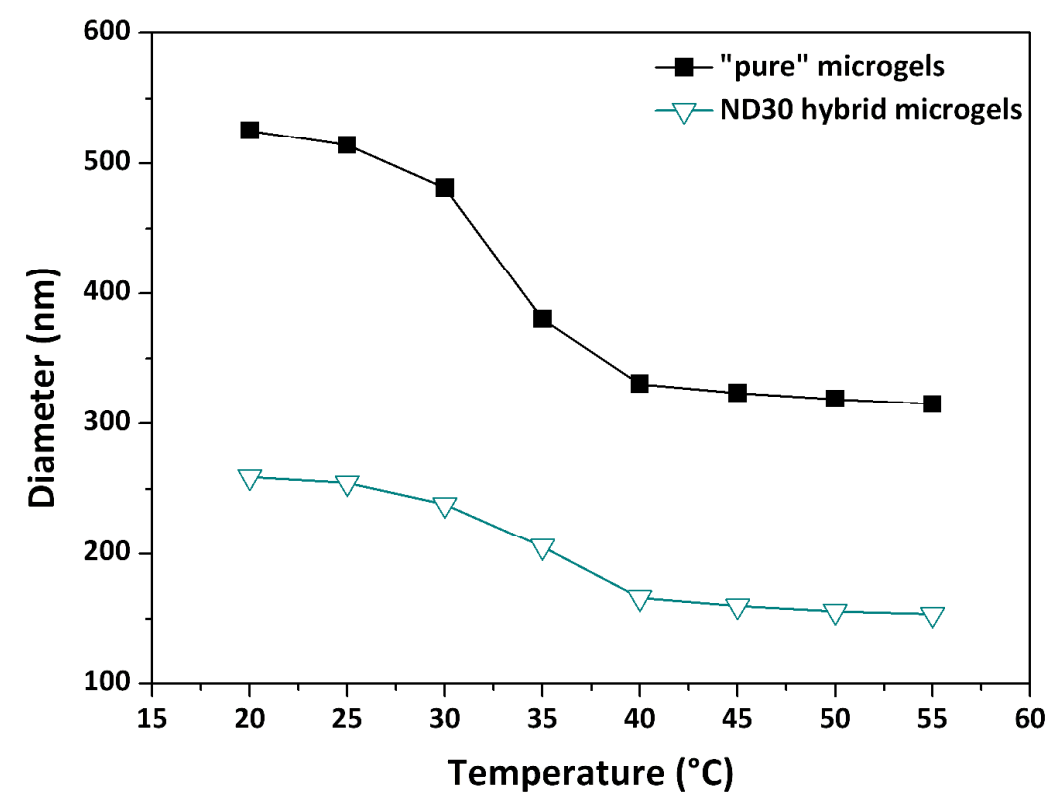

Fig.2 Evolution of the hydrodynamic diameters of « pure » pNIPAM microgels and ND30 hybrid microgels according to the temperature

pNIPAM microgels are known to undergo morphological modification according to an external stimuli like the temperature [42], so-called smart properties of the microgels. DLS 
temperature trends between 20 and $55{ }^{\circ} \mathrm{C}$ were thus realized. The variation of the hydrodynamic diameters with respect to the temperature is shown in the fig. 2 . Temperature trends of "pure" and ND30 hybrid microgels are compared. For "pure" microgels, diameters of 525 and $315 \mathrm{~nm}$ were recorded at 20 and $55^{\circ} \mathrm{C}$ respectively. From these minimum and maximum diameters, a swelling ratio can be calculated, which is defined as the ratio between the particle volume in collapsed and swollen state:

$$
\alpha=\frac{V_{\text {collapsed }}}{V_{\text {swollen }}}=\left(\frac{R_{h}\left(55^{\circ} \mathrm{C}\right)}{R_{h}\left(20^{\circ} \mathrm{C}\right)}\right)^{3}
$$

$\mathrm{R}_{\mathrm{h}}\left(55^{\circ} \mathrm{C}\right)$ is the hydrodynamic radius at $55^{\circ} \mathrm{C}$, and $\mathrm{R}_{\mathrm{h}}\left(20^{\circ} \mathrm{C}\right)$ is the hydrodynamic radius in the fully swollen state at $20^{\circ} \mathrm{C}$.

"Pure" pNIPAM microspheres exhibit a swelling ratio of 0.22 . For synthesis in which NDs were added, temperature trend shows a decrease of the diameters from 259 to $154 \mathrm{~nm}$ at 20 and $55^{\circ} \mathrm{C}$ respectively. The corresponding swelling ratio exhibits a value of 0.21 , very close to the value obtained from "pure" microgels. At the same time, the transition between the swollen and the collapsed states occurs roughly in the same temperature range, around $32^{\circ} \mathrm{C}$. Therefore, if the addition of NDs in the reaction mixture induces a size modification of the resulting microgels, it does not affect significantly their swelling properties. A collapsing of the microsphere with increased temperature still occurs, and in a reversible manner. Smart properties of pNIPAM thus remain unaffected even when NDs are introduced in the reaction media, only with a size offset. 


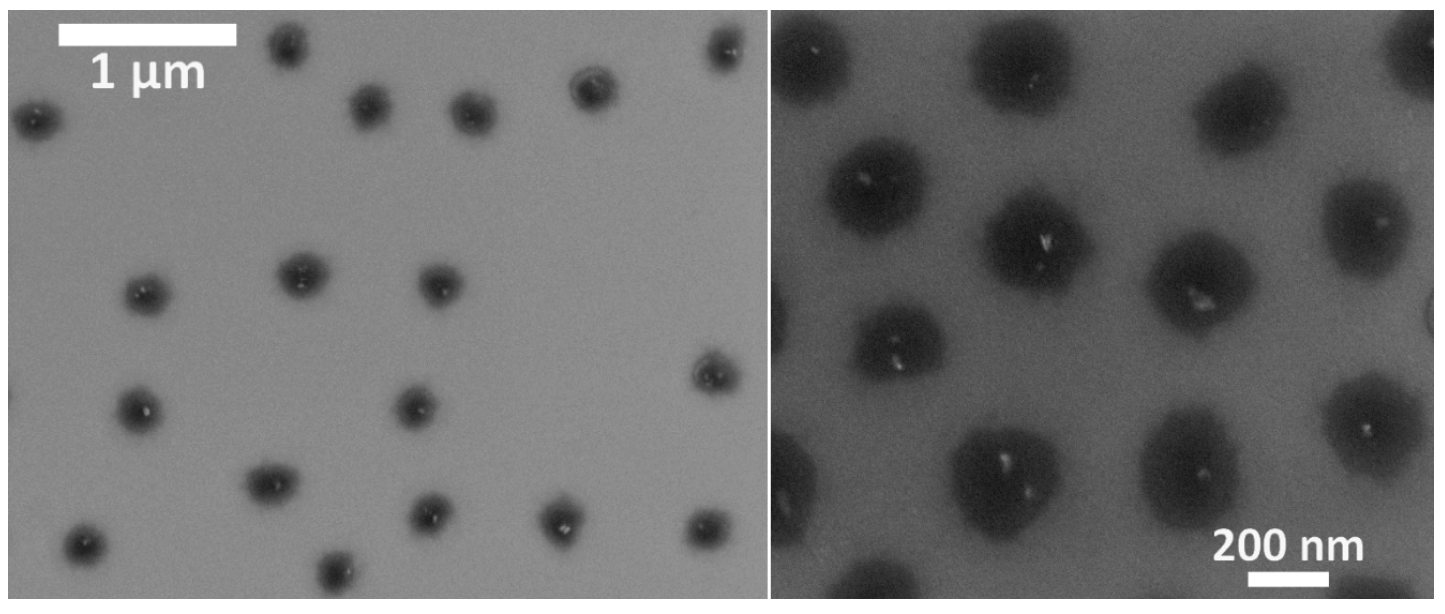

Fig.3 SEM pictures of ND30 hybrid microgels deposited on a Si substrate

SEM characterizations were performed to check the structure of the microspheres. Fig. 3 shows the SEM pictures of microgels resulting from the addition of ND30. Considering only the general morphology, the addition of NDs in the reaction medium does not affect the spherical shape of the pNIPAM structures, which consist of independent and quasi monodispersed objects. Compared to DLS measurements, the diameter undergoes a decrease, with a mean value of $210 \mathrm{~nm}$ from the SEM pictures, which is attributed to the vacuum environment of the SEM chamber. On the magnification, brighter spots can be revealed within the microspheres, which can be attributed to NDs. It is important to note that these brighter spots are only colocalized within microspheres, and not everywhere on the substrate. This point supports that, at the deposition of the solution drop, NDs were attached to the microspheres and not only left in the suspension among them. Obviously, at this stage, one could not assert that all microspheres are filled with NDs, as brighter spots assigned to NDs are not observable in all microspheres. However, HPHT nanodiamonds used in this study are polydispersed, with NDs of sizes ranging from few nanometers to tens of nanometers. One could not exclude that the smallest particles are hidden by the polymeric shell. More insights on the presence of NDs in microspheres will be given in the second part of this article, after 
polymer removal. Nevertheless, these SEM pictures confirm that hybrid objects were synthesized, consisting in NDs/pNIPAM systems.

\begin{tabular}{|c|c|c|c|c|c|}
\hline $\begin{array}{c}\text { Sample } \\
\text { name }\end{array}$ & $\begin{array}{l}\text { NDs } \\
\text { diameter } \\
(\mathrm{nm})\end{array}$ & $\begin{array}{c}\text { NDs } \\
\text { Concentration } \\
(\mu \mathrm{g} / \mathrm{mL})\end{array}$ & $\begin{array}{c}\text { Microgel } \\
\text { diameter at } \\
20^{\circ} \mathrm{C}(\mathrm{nm})\end{array}$ & $\begin{array}{c}\text { Microgel } \\
\text { diameter at } \\
55^{\circ} \mathrm{C}(\mathrm{nm})\end{array}$ & $\begin{array}{c}\text { Swelling } \\
\text { ratio }\end{array}$ \\
\hline- & - & 0 & 525 & 315 & 0.22 \\
\hline ND30-a & 30 & 17 & 362 & 201 & 0.17 \\
\hline ND30-b & 30 & 50 & 259 & 154 & 0.21 \\
\hline ND30-C & 30 & 500 & 191 & 111 & 0.20 \\
\hline ND100-a & 100 & 50 & 467 & 257 & 0.17 \\
\hline
\end{tabular}

Table 1: Maximal, minimal diameters and swelling ratios of microgels according to the diameters and the concentration of the NDs introduced during the synthesis.

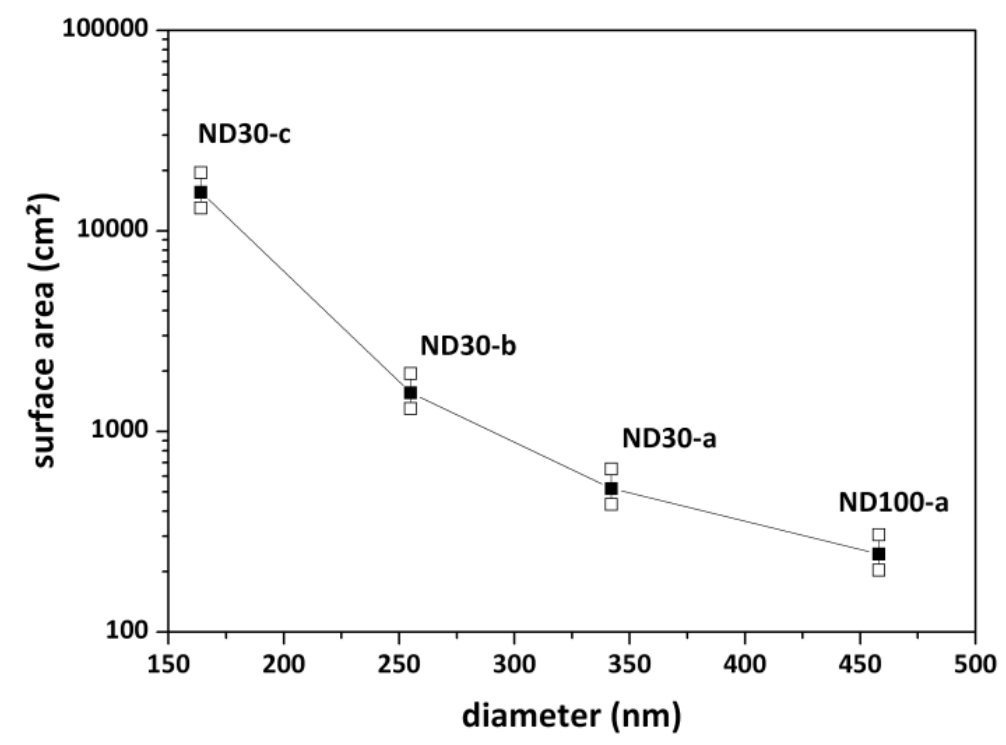

Fig.4 Estimated surface area of NDs introduced in each synthesis according to the diameter of the resulting microsphere in swollen state.

As NDs are introduced in the reaction mixture through the aqueous phase, without any functionalization, the subsequent chemical mechanisms involved in the synthesis of the 
hybrid object have to be resolved. To gain further insights on the interactions between the NDs and the monomers, additional synthesis were performed. The effect of three different concentrations (from 17 to $500 \mu \mathrm{g} / \mathrm{mL}$ ) and two different mean diameters (30 and $100 \mathrm{~nm}$ ) of NDs were studied. Concentrations, diameters at $20^{\circ} \mathrm{C}$ and $55^{\circ} \mathrm{C}$ and swelling ratios of the resulting microgels are reported in the table 1 .

Remarkable information concerns the evolution of the size of the microgels with respect to the NDs initial concentration. If the swelling ratio is kept almost constant, both swollen and collapsed states of the microgels undergo a strong decrease of their diameter for higher NDs concentration. Besides, when the mean diameter of the NDs introduced in the reaction mixture is raised from 30 to $100 \mathrm{~nm}$, at similar concentration, the diameter of the resulting microgels is also increased. Fig. 4 reports the diameter $\left(\right.$ at $\left.20^{\circ} \mathrm{C}\right)$ of each microgel according to the developed surface area of the NDs introduced in the synthesis. These surface areas were calculated using a very simple approach, considering a spherical particle, their mean diameter and their concentration in the solution. Note that the polydispersity of the NDs was taking into account and represented by the error bars. An interesting point concerns the $100 \mathrm{~nm}$ nanoparticles. With an initial concentration of $50 \mu \mathrm{g} / \mathrm{mL}$ in the aqueous phase, calculations show that they finally develop a surface area lower than any $30 \mathrm{~nm}$ particles, whatever their concentration. At the same time, resulting microgels exhibit a larger diameter than those loaded with $30 \mathrm{~nm}$, which is in agreement with the trend observed for the different concentration of the $30 \mathrm{~nm}$ NDs. Thus, it confirms that the size of the resulting hybrid microspheres depends on the total surface area of the NDs introduced in the reaction mixture, and not only on their size.

As a first approach, it can be suggested that polymerization starts from the surface of the NDs, as in core-shell structure. A proposed mechanism could be based on a seed precipitation polymerization, as already described in the literature. Deng and coworkers reported the 
formation of pNIPAM spheres around a silica core, through the addition of $\mathrm{C}=\mathrm{C}$ bonds on the surface of the silica cores[59]. Here, no specific functionalization was applied to NDs, but they natively carry on their surface carboxylic groups as well as a mix of $\mathrm{sp}^{2}$ and $\mathrm{sp}^{3}$ carbons in aliphatic structures[60]. The contribution these $\mathrm{C}=\mathrm{C}$ bonds may play in the polymerization can be significant, as well as the interaction between the carboxylic groups and the NIPAM monomer. Another experimental observation comes in support of this hypothesis, which concerns the light opalescence during the synthesis. The appearance of the opalescence somehow reveals when the microgels start to grow in the suspension. Here, depending on the total NDs surface area introduced in the aqueous phase, a delay of the opalescence appearance has been observed, which can reach $4 \mathrm{~h}$ for the maximal concentration of NDs, i.e. the largest surface area, compared to few minutes for $100 \mathrm{~nm}$ NDs, i.e. the lower surface area. Therefore, a preliminary step of adsorption/grafting of the reagents on the NDs seems to occur, before the growth of the microsphere, directly linked to the total surface area of NDs introduced in the reaction mixture.

\subsection{Self assembled layers of NDs hybrid microgels}

Beyond the use of the hybrid microgels as "isolated objects" combining the smart properties of the pNIPAM microgels and the assets of NDs toward biological or optical applications, our novel hybrid systems can also be employed to build self-assembled layers of NDs on a surface. Fig. 5a shows a typical deposit of ND30 hybrid microgels onto a silicon substrate by dip-coating, from a diluted suspension of hybrid microgels in water, stabilized at $45^{\circ} \mathrm{C}$. These hexagonal ordered periodic loosely packed arrays result from a self-assembly of the microspheres at the air/liquid interface, occurring at the dewetting of the substrate. Such assemblies are driven by repulsive forces, resulting from electrostatic and steric interaction among the microgel, exceeding attractive forces, mainly due to capillary interactions at the 
water surface[35]. In our case, electrostatic interactions can be tuned through the zeta potential of the particles, by playing on the temperature, while steric interactions are mainly driven by the swelling or deswelling of the pNIPAM soft shell. Note that such ordered monolayers have been easily obtained on large areas of several hundreds of square micrometers. From the magnification (insert of fig. 5a), a mean separation distance between two microspheres of c.a. $500 \mathrm{~nm}$ has been calculated with an average diameter of each microspheres of $330 \mathrm{~nm}$. The same sample was then exposed to $\mathrm{H}_{2} / \mathrm{CH}_{4} \mathrm{CVD}$ plasma at 750 ${ }^{\circ} \mathrm{C}$ for few minutes, in order to burn all the polymeric phases. Indeed, we already showed that such CVD treatment is efficient to remove polymeric layers on NDs [21,23]. Note that in these conditions, NDs are preserved from any etching, a weak growth (few nm) can even occur. As shown on Fig. 5b, after such plasma treatment, the organized microspheres have given the place to organized NDs (bright spots), surrounded by a dark disk, which can be reasonably attributed to a mark left by the microspheres on the silicon substrate when burned away. The hexagonal order of the periodic loosely packed arrays is retained, with a similar separation distance between the objects. From the magnification (insert of Fig. 5b), it appears that almost all microspheres contained at least one ND, which confirms their encapsulation in pNIPAM during the synthesis. Furthermore, the removal of the polymer matrix reveals the scattered size distribution of the encapsulated NDs, ranging from few nanometers to tens of nanometers, in agreement with DLS measurements (see table 2). This explains the difficulty we had to observe NDs in all microspheres (see fig. 3), the smallest being hidden by the polymer layer. 

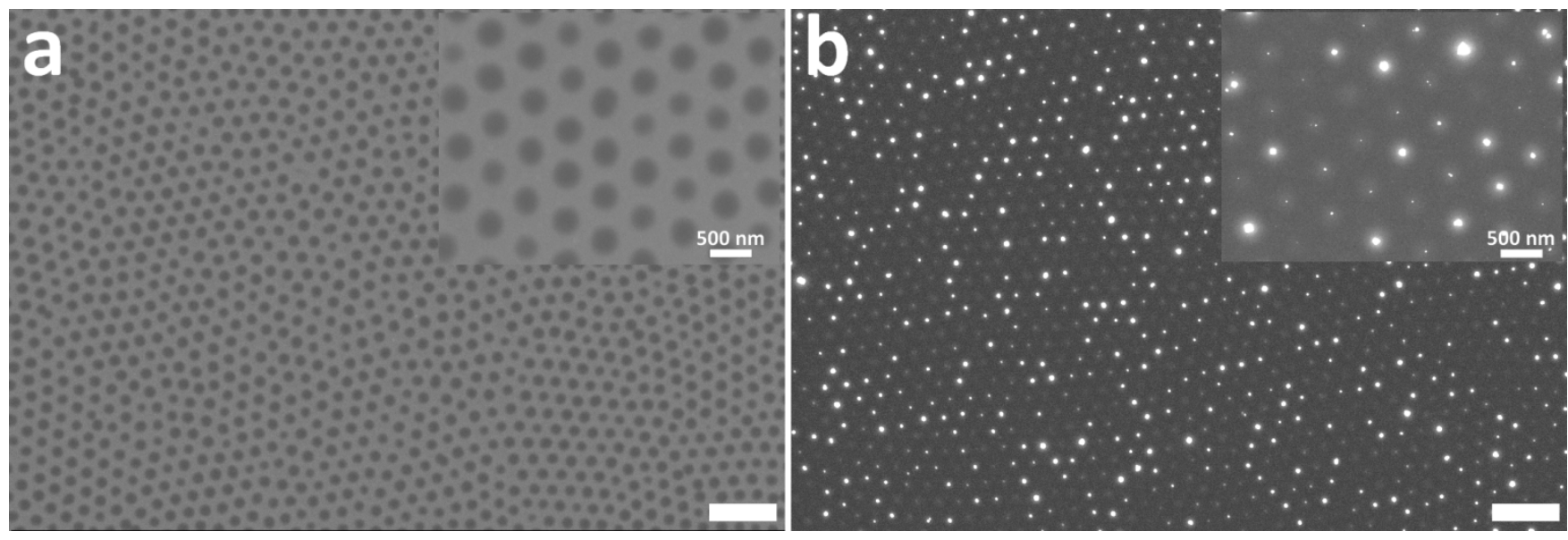

Figure 5 SEM pictures of periodic loosely packed arrays of ND30 hybrid microgels (a) before and (b) after CVD plasma. Scale bare : $1 \mu \mathrm{m}$

The deposition parameters during the dip-coating, as the temperature, the concentration of the suspension, or the dewetting velocity are key parameters to control the architecture of the selfassembled monolayer. For instance, Fig. 6 exhibits close packed (Fig. 6a) and periodic loosely packed (Fig. 6b) arrays obtained from the same suspension of ND30 hybrid microgels. To achieve these close packed arrays, temperature of the suspension during the deposition was decreased to $20^{\circ} \mathrm{C}$, against $50^{\circ} \mathrm{C}$ for periodic loosely packed, reducing the overall surface charge of each particle and enhancing their swelling, and the suspension was 20 times more concentrated. Zeta potential measurements reported a strong decrease of the absolute value of the particles charge when reducing the temperature, from $-32 \mathrm{mV}$ at $50^{\circ} \mathrm{C}$ to $-18 \mathrm{mV}$ at $20^{\circ} \mathrm{C}$, thus minimizing the electrostatic repulsion forces between the particles. Consequently, close packed arrays were realized with a separation distance between centers of two microspheres reduced from c.a. $600 \mathrm{~nm}$ to c.a. $335 \mathrm{~nm}$. At the same time, all microspheres lie in contact to each other, and the diameter of each microsphere has been reduced from c.a. $260 \mathrm{~nm}$ to c.a. $159 \mathrm{~nm}$ for loosely packed and closed packed arrays respectively. It can also be noticed that for loosely packed arrays, a decrease of $5^{\circ} \mathrm{C}$ of the suspension temperature during the deposition results in a reduced separation distance between microspheres of $100 \mathrm{~nm}$, if we 
compare Fig. 5a and Fig. 6b. This illustrates the versatility of the method to control the separation distance between the microspheres organized in $2 \mathrm{D}$ arrays.
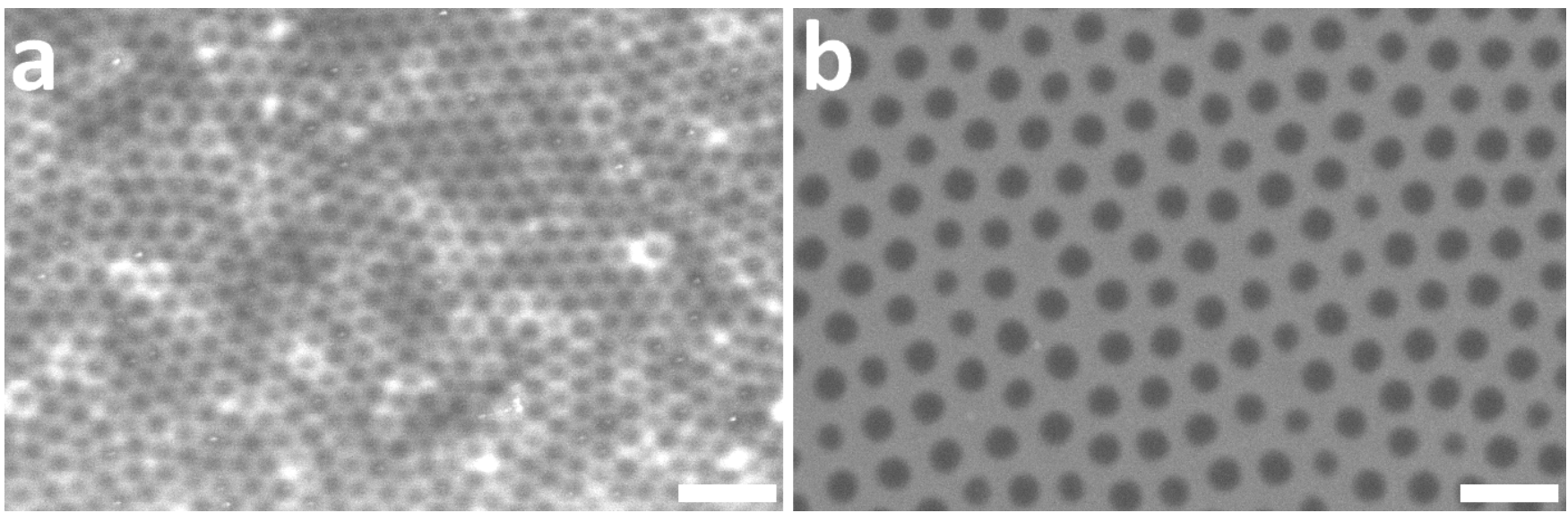

Figure 6: SEM pictures of (a) close packed (b) periodic loosely packed arrays of ND30 hybrid microgels. Scale bare : $1 \mu \mathrm{m}$

\section{Conclusions}

In this study, we report on the synthesis of nanodiamonds hybrid microgels, characterized by DLS and SEM. These new hybrid microgels exhibit similar smart properties than "pure" pNIPAM microspheres, as the reversible collapsing of the structures when raising the temperature, with almost equivalent swelling ratios and transition temperatures. Reaction mechanisms at the origin of the loading the NDs is still under debate, but a correlation between the surface areas of the NDs introduced in the reaction media and the size of the resulting microspheres suggests a precipitation polymerization starting from the surface of the NDs, through a prior adsorption of the reagents on the NDs surface.

Such hybrid microspheres are very promising for different fields of applications. The possibility to achieve 2D organized arrays of nanodiamonds through the use of pNIPAM 
encapsulation open up the doors to cost-limited nanoscaled NDs organization on a substrate with a great flexibility in term of dimensions, toward useful technological or optical applications. For instance, such assemblies can constitute a first step toward a bottom-up elaboration of diamond-based devices, from simple mask lithography to 2D or 3D systems toward catalysis, biological or optical uses. The synthesis of these novel ND-based hybrid objects is thus very promising for the realization of innovative diamond-based devices.

\section{Acknowledgements}

The author would like to thank the financial support from the French Atomic Commission.

\section{References}

[1] S.-J. Yu, M.-W. Kang, H.-C. Chang, K.-M. Chen, Y.-C. Yu, J. Am. Chem. Soc. 127 (2005) 17604-17605.

[2] Y.-R. Chang, H.-Y. Lee, K. Chen, C.-C. Chang, D.-S. Tsai, C.-C. Fu, T.-S. Lim, Y.-K. Tzeng, C.-Y. Fang, C.-C. Han, H.-C. Chang, W. Fann, Nat Nano 3 (2008) 284-288.

[3] A.D. Greentree, I. Aharonovich, S. Castelletto, M.W. Doherty, L.P. McGuinness, D.A. Simpson, Opt. Photon. News 21 (2010) 20-25.

[4] L.A. Stewart, Y. Zhai, J.M. Dawes, M.J. Steel, J.R. Rabeau, M.J. Withford, Optics Express 17 (2009) 18044.

[5] L.P. McGuinness, Y. Yan, A. Stacey, D.A. Simpson, L.T. Hall, D. Maclaurin, S. Prawer, P. Mulvaney, J. Wrachtrup, F. Caruso, R.E. Scholten, L.C.L. Hollenberg, NATURE NANOTECHNOLOGY 6 (2011) 358-363.

[6] L.-C.L. Huang, H.-C. Chang, Langmuir 20 (2004) 5879-5884.

[7] Y. Liu, Z. Gu, J.L. Margrave, V.N. Khabashesku, Chemistry of Materials 16 (2004) 3924-3930.

[8] T. Petit, J.-C. Arnault, H.A. Girard, M. Sennour, P. Bergonzo, Physical Review B 84 (2011) 233407.

[9] H.A. Girard, J.C. Arnault, S. Perruchas, S. Saada, T. Gacoin, J.-P. Boilot, P. Bergonzo, Diamond Relat. Mater. 19 (2010) 1117-1123. 
[10] H.A. Girard, T. Petit, S. Perruchas, T. Gacoin, C. Gesset, J.C. Arnault, P. Bergonzo, Physical Chemistry Chemical Physics : PCCP 13 (2011) 11517-11523.

[11] A. Alhaddad, M.-P. Adam, J. Botsoa, G. Dantelle, S. Perruchas, T. Gacoin, C. Mansuy, S. Lavielle, C. Malvy, F. Treussart, J.-R. Bertrand, Small (2011) n/a-n/a.

[12] M. Chen, X.Q. Zhang, H.B. Man, R. Lam, E.K. Chow, D. Ho, The Journal of Physical Chemistry Letters 1 (2010) 3167-3171.

[13] S.A. Dahoumane, M.N. Nguyen, A. Thorel, J.-P. Boudou, M.M. Chehimi, C. Mangeney, Langmuir 25 (2009) 9633-9638.

[14] H. Huang, E. Pierstorff, E. Osawa, D. Ho, Nano Letters 7 (2007) 3305-3314.

[15] E.K. Chow, X.-Q. Zhang, M. Chen, R. Lam, E. Robinson, H. Huang, D. Schaffer, E. Osawa, A. Goga, D. Ho, Science Translational Medicine 3 (2011) 73ra21.

[16] X.-Q. Zhang, M. Chen, R. Lam, X. Xu, E. Osawa, D. Ho, ACS Nano 3 (2009) 260916.

[17] M. Chen, E.D. Pierstorff, R. Lam, S.-Y. Li, H. Huang, E. Osawa, D. Ho, ACS Nano 3 (2009) 2016-2022.

[18] K.-K.L. and W.-W.Z. and C.-C.W. and Y.-C.C. and C.-L.C. and Y.-S.L. and C.C. and J.-I. Chao, Nanotechnology 21 (2010) 315106.

[19] K. Goldberg, A. Krueger, T. Meinhardt, W. Kroutil, B. Mautner, A. Liese, Tetrahedron: Asymmetry 19 (2008) 1171-1173.

[20] O.A. Williams, O. Douh $\square$ ret, M. Daenen, K. Haenen, E. Osawa, M. Takahashi, Chem. Phys. Lett. 445 (2007) 255-258.

[21] E. Scorsone, S. Saada, J.C. Arnault, P. Bergonzo, J. Appl. Phys. 106 (2009) 14908.

[22] O. Shenderova, S. Hens, G. McGuire, Diamond and Related Materials 19 (2010) 260267.

[23] H.A. Girard, S. Perruchas, C. Gesset, M. Chaigneau, L. Vieille, J.-C. Arnault, P. Bergonzo, J.-P. Boilot, T. Gacoin, ACS Applied Materials \& Interfaces 1 (2009) 27382746.

[24] J. Hees, A. Kriele, O. a. Williams, Chemical Physics Letters 509 (2011) 12-15.

[25] H.A. Girard, E. Scorsone, S. Saada, C. Gesset, J.C. Arnault, S. Perruchas, L. Rousseau, S. David, V. Pichot, D. Spitzer, P. Bergonzo, Diamond and Related Materials 23 (2012) 83-87.

[26] E. Chevallier, E. Scorsone, H.A. Girard, V. Pichot, D. Spitzer, P. Bergonzo, Sensors and Actuators B: Chemical 151 (2010) 191-197. 
[27] H. Huang, E. Pierstorff, E. Osawa, D. Ho, ACS Nano 2 (2008) 203-212.

[28] A. Thalhammer, R.J. Edgington, L.A. Cingolani, R. Schoepfer, R.B. Jackman, Biomaterials 31 (2010) 2097-2104.

[29] J. Hu, X. Lu, J.S. Foord, Electrochemistry Communications 12 (2010) 676-679.

[30] K.B. Holt, C. Ziegler, D.J. Caruana, J. Zang, E.J. Millan-Barrios, J. Hu, J.S. Foord, Phys. Chem. Chem. Phys. 10 (2008) 303-310.

[31] C. Lu, Y. Li, S. Tian, W. Li, J. Li, C. Gu, Microelectronic Engineering 88 (2011) 2319-2321.

[32] X.M. Lin, H.M. Jaeger, C.M. Sorensen, K.J. Klabunde, The Journal of Physical Chemistry B 105 (2001) 3353-3357.

[33] O.D. Velev, T.A. Jede, R.F. Lobo, A.M. Lenhoff, Nature 389 (1997) 447-448.

[34] P.M. Tessier, O.D. Velev, A.T. Kalambur, J.F. Rabolt, A.M. Lenhoff, E.W. Kaler, Journal of the American Chemical Society 122 (2000) 9554-9555.

[35] M. Horecha, V. Senkovskyy, A. Synytska, M. Stamm, A.I. Chervanyov, A. Kiriy, Soft Matter 6 (2010) 5980-5992.

[36] Y. Lu, M. Drechsler, Langmuir 25 (2009) 13100-13105.

[37] M. Ballauff, Y. Lu, Polymer 48 (2007) 1815-1823.

[38] R. Pelton, Advances in Colloid and Interface Science 85 (2000) 1-33.

[39] D. Duracher, A. Elaïssari, F. Mallet, C. Pichot, Langmuir 16 (2000) 9002-9008.

[40] R.F.S. Freitas, E.L. Cussler, Chemical Engineering Science 42 (1987) 97-103.

[41] M.J. Serpe, C.D. Jones, L.A. Lyon, Langmuir 19 (2003) 8759-8764.

[42] S. Pankasem, J.K. Thomas, M.J. Snowden, B. Vincent, Langmuir 10 (1994) 30233026.

[43] T. Hoare, R. Pelton, Langmuir 22 (2006) 7342-7350.

[44] M. Shibayama, F. Ikkai, S. Inamoto, S. Nomura, C.C. Han, The Journal of Chemical Physics 105 (1996) 4358-4366.

[45] S.B. Quint, C. Pacholski, Soft Matter 7 (2011) 3735-3738.

[46] L. Lu, A. Eychmüller, Accounts of Chemical Research 41 (2008) 244-253.

[47] K. Wu, L. Shi, W. Zhang, Y. An, X.-X. Zhu, Journal of Applied Polymer Science 102 (2006) 3144-3148. 
[48] M. Karg, T. Hellweg, Journal of Materials Chemistry 19 (2009) 8714.

[49] N. Shamim, L. Hong, K. Hidajat, M.S. Uddin, Colloids and Surfaces. B, Biointerfaces 55 (2007) 51-8.

[50] M. Das, N. Sanson, D. Fava, E. Kumacheva, Langmuir 23 (2006) 196-201.

[51] H.H. Park, T.R. Lee, Journal of Nanoparticle Research 13 (2010) 2909-2918.

[52] K. Zhang, J. Ma, B. Zhang, S. Zhao, Y. Li, Y. Xu, W. Yu, J. Wang, Materials Letters 61 (2007) 949-952.

[53] O. Tagit, N. Tomczak, A. Jafarpour, D. Jańczewski, M.Y. Han, G.J. Vancso, J.L. Herek, Nanotechnology 22 (2011) 265701.

[54] A.Z. Pich, H.-J.P. Adler, Polymer International 56 (2007) 291-307.

[55] A. Pich, J. Hain, Y. Lu, V. Boyko, Y. Prots, H.-J. Adler, Macromolecules 38 (2005) 6610-6619.

[56] A. Barras, J. Lyskawa, S. Szunerits, P. Woisel, R. Boukherroub, Langmuir (2011).

[57] R.H. Pelton, P. Chibante, Colloids and Surfaces 20 (1986) 247-256.

[58] B.R. Saunders, B. Vincent, Advances in Colloid and Interface Science 80 (1999) 1-25.

[59] Y.H. Deng, W.L. Yang, C.C. Wang, S.K. Fu, Advanced Materials 15 (2003) 17291732.

[60] M. Chaigneau, H.A. Girard, J.-C. Arnault, R. Ossikovski, MRS Proceedings 1282 (2011). 